\title{
Probiotics and Reduction of the Inflammatory Response for Prevention of Preeclampsia
}

\section{Probióticos y Reducción de la Respuesta Inflamatoria para la Prevención de la Preeclampsia}

XVIII International Seminar on Health, Food and Human Nutrition

Corresponding Author:

Iván Enrique Naranjo Logroño

naranjometropolitana@hotmail.com

Published: 9 September 2021

Production and Hosting by

Knowledge E

(a) Iván Enrique Naranjo Logroño et al. This article is distributed under the terms of the Creative Commons Attribution License, which permits unrestricted use and redistribution provided that the original author and source are credited.

\author{
Iván Enrique Naranjo Logroño ${ }^{1}$, Anthony Alfonso Naranjo Coronel ${ }^{2}$, Cynthia \\ Johana Navarro Rivadeneira ${ }^{1}$, and Erika Alejandra Romero Minaya ${ }^{1,3}$ \\ ${ }^{1}$ Carrera de Medicina, Facultad de Salud Pública, ESPOCH, Riobamba, Chimborazo, Ecuador \\ ${ }^{2}$ Médico Cirujano, COLPOMED Centro, Hospital del Día, Riobamba, Chimborazo, Ecuador \\ ${ }^{3}$ Hospital Provincial General Docente de Riobamba, Riobamba, Ecuador
}

\section{Abstract}

Introduction: In the field of medicine and obstetrics, the most common hypertensive disorders are preeclampsia and it constitutes a great question that has not yet been completely resolved. Objective: To identify the effectiveness of the use of probiotics and the reduction of the inflammatory response in the prevention of pre-eclampsia. Method: This search took place in large databases both globally and locally, being Elsevier, La Reference, Scielo, Pubmed, Google Scholar, Redalyc, Scopus, among others, where probiotic and pre-eclampsia terms were found. Results: 37 articles were found which were preselected, after correct appreciation 32 articles were selected and 5 articles were discarded, the selected ones met the inclusion and exclusion criteria, therefore they are related to the topic and objective of the present study review. Discussion: The epithelial cells of the intestine produce TNF- $\alpha$, once mixtures of different strains of probiotics are made, causing the immune response to be generated, especially if consumed before pregnancy or in early pregnancy and continued throughout of the same. the administration is safe and has a protective role in pre-eclampsia, gestational diabetes, vaginal infections, maternal, infant, and subsequent weight gain. Conclusion: The preventive effect occurs as long as probiotics are administered mainly in the second and third trimesters of pregnancy, although the consumption of probiotics before these results in a much greater benefit, and is even more potent if probiotic mixtures are made.

Keywords: probiotics, preeclampsia, hypertensive disorder.

\section{Resumen}

Introducción: En el campo de la medicina y obstetricia, los trastornos hipertensivos más comunes son preeclampsia y compone una gran interrogante que aún no está resuelta completamente. Objetivo: Identificar la efectividad del uso de probióticos y la reducción de la respuesta inflamatoria en la prevención de la preeclampsia. Método: Esta búsqueda tuvo lugar en grandes bases de datos tanto a nivel mundial como local, siendo Elsevier, La Referencia, Scielo, Pubmed, Google Académico, Redalyc, Scopus, entre otras, donde se encontraron términos de probióticos y preeclampsia. Resultados: Se encontró 37 artículos los cuales fueron preseleccionados, luego de la correcta apreciación se seleccionaron 32 artículos y se descartaron 5 artículos, los seleccionados cumplían con los criterios inclusión y de exclusión por lo tanto van en relación con el tema y objetivo del presente estudio de revisión. Discusión: Las células epiteliales del intestino producen TNF- $\alpha$, una vez que se realicen mezclas de 
distintas cepas de probióticos, haciendo que se genere la respuesta inmunitaria, en especial si se consume antes del embarazo o en el embarazo temprano y continua a lo largo del mismo. La administración es segura y tiene un papel protector en la preeclampsia, diabetes gestacional, infecciones vaginales, aumento de peso materno, infantil, y posteriores. Conclusión: El efecto preventivo se da siempre y cuando se administre probióticos principalmente en el segundo y tercer trimestre de embarazo, aunque el consumo de probióticos con anterioridad a estos resulta de mucho mayor beneficio, y se potencia aún más si se realizan mezclas de probióticos.

Palabras Clave: probióticos, preeclampsia, trastorno hipertensivo.

\section{Introducción}

El embarazo es un estado que implica muchas adaptaciones fisiológicas que permitirán albergar al nuevo ser en formación, dicha etapa no está exenta de complicaciones. Reportes de la OMS, en todo el mundo, a diario mueren aproximadamente 830 mujeres embarazadas por complicaciones relacionadas con el mismo embarazo o con el parto [1].

Los trastornos hipertensivos constituyen uno de los problemas más intrigantes, y aún no resuelto en el campo de la medicina y obstetricia, de acuerdo con las estadísticas disponibles hoy en día se calcula que de un 2 a un $8 \%$ los embarazos a nivel mundial son complicados y con ello más de un $20 \%$ de muertes maternas en todo el mundo se dan debido a la preeclampsia [2, 3].

A pesar de que la preeclampsia representa un riesgo potencial de muerte para la madre, también repercute en el desarrollo normal del feto, puesto que se disminuye el suministro de sangre, mecanismo por el cual el feto recibe nutrientes, con lo cual no existirá este último, a esta condición se le conoce como restricción del crecimiento intrauterino (RCIU). Alrededor del mundo se estima que cada año nacen 30 millones de bebés con $\mathrm{RCIU}$, de ellos el 15\% (4,5 millones) son repercusión de preeclampsia [4]. Este trastorno es más frecuente en aquellos países que están aún en vías de desarrollo, Ecuador es uno de ellos y en el 2010 la preeclampsia-eclampsia fue una de las principales causas de morbimortalidad materna y perinatal [5].

La fisiopatología de la preeclampsia se da por la implantación placentaria anómala lo que conlleva a un estado de hipoxia, y por ende se produce la respuesta inflamatoria materna, sin embargo esta causa aún no está del todo clara, en estudios ya realizados aluden que poseen características inmunológicas, puesto que en el sistema inmune existe una gran variabilidad de genes, estos tienen la función de codificar las moléculas que forman el complejo de histocompatibilidad por lo tanto al sufrir esta modificación los receptores de las células llamadas asesinas naturales, modifican la placentación generando cambios en la misma [6].

Además de esto el incremento de costos de un parto con riesgos maternos neonatales son mayores, que aquellos partos que suceden si eventualidades. El impacto de los trastornos hipertensivos en el embarazo es muy grave puesto que afecta social y económicamente en el desarrollo de un país [7]. El tratamiento de este trastorno en 
la actualidad es el nacimiento del bebe y la expulsión de la placenta, puesto que la medicación utilizada solo nos permite aumentar las posibilidades de supervivencia del recién nacido y con el uso de anticonvulsivantes para la madre, se pretende evitar efectos adversos, por lo tanto el diagnostico oportuno y la prevención son los mejores pilares, por lo cual surge la pregunta ¿tienen los probióticos mecanismos para reducir la respuesta inflamatoria y con ello evitar la preeclampsia? [8].

Con el presente estudio se pretende: analizar las diversas investigaciones sobre el efecto de los probióticos en la prevención de la preeclampsia y de esa manera contribuir con la literatura médica de Latinoamérica y del mundo que se enfocan en la alimentación materna como base para que la mujer durante la etapa de embarazo tenga un sistema inmunológico sano; el consumo de probióticos, que son microorganismos productores de ácido láctico con lo cual disminuyen la permeabilidad intestinal a bacterias patógenas, incrementa la producción de los linfocitos $T$ helper, y de inmunoglobulina A secretora, así influyen en las respuestas inmunitarias, mediadas por el tejido linfoide asociado al intestino [9].

\section{Metodología}

\subsection{Diseño: En el siguiente artículo de revisión, los criterios meta analíticos no forman parte del mismo}

Estrategia de búsqueda: Para tomar en cuenta los criterios de inclusión, las investigaciones que enfocaron la atención en probióticos y reducción de la respuesta inflamatoria para la prevención de la preeclampsia han sido aceptadas; los estudios publicados desde el año 2005, han servido de base; posteriormente, se incluyeron solo artículos originales. Al momento de realizar la exclusión se toma en cuenta los artículos que contengan más variables fenomenológicas o búsquedas sin resultados en su contenido y artículos sin texto selecto. Cuando se realizó la preselección, los artículos, fueron sometidos a un segundo proceso de indagación, sobresaliendo los que contenían información acerca de: probióticos, respuesta inflamatoria, prevención de preeclampsia, logrando conseguir artículos originales de relevancia, que, en su gran mayoría fueron experimentales.

Esta búsqueda tuvo lugar en grandes bases de datos tanto a nivel mundial como local, siendo Elsevier, La Referencia, Scielo, Pubmed, Google Académico, Redalyc, Scopus, entre otras, donde se encontraron términos de probióticos y preeclampsia. Entre diciembre 2019-febrero 2020, se investigó toda la bibliografía seleccionada. Se utilizó palabras clave en todas las bases ya mencionadas, siendo estas: Probióticos, preeclampsia, además de utilizar términos boleanos 'and' y 'or', descriptores en singular o plural e incluso buscando términos en inglés como español. 


\section{Resultados}

Selección de estudios: Al disponer de distintas bases de datos se emplearon diversos métodos para realizar la búsqueda. Como se recopiló 37 artículos, y se eliminó 5, se trabajó con los 32 artículos con la mejor información y el cumplimiento de los criterios, pues los descartados no se ajustaban del todo con el objetivo de la revisión.

\section{Discusión}

Probióticos: Son microorganismos vivos que están destinados a mantener o mejorar el microbiota normal, a diferencia de los prebióticos, que son alimentos que sirven de nutrientes para la microbiota. Se encuentra principalmente en alimentos como el yogurt y el chucrut (col blanca fermentada con vinagre) [10].

Preeclampsia: Es una complicación asociada al embarazo y relativamente común, caracteriza por la presencia de cierto signos y síntomas como la hipertensión arterial, proteínas en la orina y daño a órganos, siendo más frecuente el hígado y riñones. Ocurre a partir de la semana 20 de embarazo tras tener una presión arterial hasta ese entonces, normal. Existen diferentes factores de riesgo para que aparezca, como, ser madre primeriza, embarazo previo cursado con esta patología, embarazos múltiples, edad materna menor de 20 o mayor de 40 años, obesidad o antecedentes de enfermedad renal, entre otros. Además, se clasifica en leve y severa, dependiendo la sintomatología [11].

La etiología de la preeclampsia se cree que se debe a un fallo en la placentación. Al inicio del desarrollo embrionario la nutrición está dada por secreciones de las glándulas endometriales, conocido como nutrición histiotrófica (nutrición de sustratos disponibles en útero), posteriormente el trofoblasto invade los tejidos maternos y se forman lagunas que después se unen para crear el espacio intervelloso, la apertura de las arterias espirales en dicho espacio permite el cambio de nutrición de tipo histiotrófica a hematotrófica. Cuando el proceso de placentación no es el óptimo, se produce una oxigenación inadecuada, lo que conlleva a un estado de estrés oxidativo causado por una deficiente perfusión del trofoblasto. Inmunológicamente en el embarazo normal el perfil Th (T-helper) 2 es dominante, mientras que en la preeclampsia domina el Th1. Cuando se activa el perfil Th1 (productores de Inter Leucina-2, Factor de necrosis tumoral interferón), las citoquinas activan las células T citotóxicas y las células NK, ampliando aún más su efecto citotóxico, causando un estado inflamatorio generalizado [12].

Al realizar cierto estudio se pudo observar que, si se realizan mezclas de cepas de probióticos, estas pueden inducir en las células epiteliales intestinales la producción de factor necrótico tumoral alfa (TNF- $\alpha$ ), quien reacciona frente a la presencia de alguna respuesta inmunitaria, actuando de manera más eficiente que al no consumir probióticos. La producción de inmunoglobulinas A y M, la producción de citoquinas cuando existe modulación de respuesta, la liberación de las quemoquinas, el desarrollo de los linfocitos $T$ reguladores y la activación de las células asesinas naturales, son activados por los probióticos al encontrarse frente a procesos inmunológicos [13]. 
Lactobacillus casei es una bacteria anaerobia Gram positiva que habita en el intestino y en la boca de los humanos y es utilizado en la elaboración de alimentos probióticos, en un estudio realizado por T Ogawa, Y Asai se demostró que en conjunción con dextrano, elevó significativamente las actividades de las células asesinas naturales (NK) en células mononucleares de bazo de ratones BALB/c en comparación con el Lactobacillus casei ssp. casei solo o con otras especies de Lactobacillus con o sin dextrano [14].

Un estudio de cohorte prospectivo mostró recientemente que la administración de una leche suplementada con probióticos durante el embarazo redujo la preeclampsia y el riesgo de parto prematuro [15].

Nordqvist $M$, Jacobsson $B$, Brantsæeter A-L, mencionan que los probióticos son microorganismos gran positivos fermentadores de carbohidratos y productores de ácido láctico. Su importancia fundamental desde el punto de vista bioterapéutico se da porque facilitan la digestión de la lactosa y equilibran el sistema inmunitario encontramos que protegen de enfermedades del aparato gastrointestinales. Se realizó también un estudio en 37.050 mujeres nulíparas y se encontró que 1851 presentaban preeclampsia leve y 550 preeclampsia grave. La ingesta de leche con probióticos durante el embarazo tardío evidenció un menor beneficio en la reducción de riesgo para la preeclampsia en comparación con las mujeres que consumieron la leche con prebióticos antes del embarazo y en el embarazo temprano, con lo cual podemos entender el efecto beneficioso tangible que tiene para el desarrollo del embarazo. Los probióticos tienen el potencial de afectar los procesos fisiopatológicos involucrados en la hipertensión, la inflamación, la función renal y la diabetes [16].

Según Garrote y Bonet, mencionan que los probióticos, mantiene la integridad del epitelio intestinal, previene los daños que pueden efectuarse por acción de microorganismos patógenos alergenos, citoquinas proinflamatorias o bien facilitan la reparación, mejorando la respuesta inflamatoria intestinal. Además, ayuda al sistema inmunológico, al activar los macrófagos locales, quienes participan en la presentación de antígenos a los linfocitos B y producen inmunoglobulina a secretora local y sistémica [17].

Pradenas Marcelo, explica en su artículo que la mayoría de probióticos contienen lactobacillus, lo cuales al estar en mayor cantidad, brindan un ambiente sano, al producir ácido láctico y al estar presentes en la flora vaginal, mantienen un ambiente inhóspito para muchas bacterias, estas especies también producen peróxido de hidrogeno que son radicales hidroxilados tóxicos y que impiden el crecimiento de bacterias patógenas, lo que resulta muy favorable para la embarazada que esta propensa a sufrir infecciones por sus cambios anatómicos [18]. Entre las bacterias que con más frecuencia se hallan en el tracto urogenital se encuentra la Chlamydia trachomatis, Trichomonas vaginalis, Escherichia coli, Enterococcus faecalis, Gardnerella vaginalis, Staphylococcus saprophyticus, Neisseria gonorrhoeae, Candida albicans y Klebsiella pneumoniae [19].

Otro artículo publicado por Espinoza, Vidaeff, Pettker y Simhan sobre el consumo de probiótico de leche durante el embarazo y su ayuda en la prevención de preeclampsia, señalan la importancia de una alimentación materna preconcepcional rica en vegetales favorecida con un equilibrado ambiente creado por dichos microrganismos probióticos [20]. 
En la Guía de práctica clínica del Ministerio de Salud Pública sobre los trastornos hipertensivos del embarazo destaca que la preeclampsia y eclampsia son las primeras causas de muerte materna desde el año 2006 a 2014, y representan el 27,53\% de todas las muertes maternas, por lo que representa una patología de alto riesgo en nuestro medio, además se relaciona con muchas complicaciones tanto maternas como perinatales [21].

Existe una respuesta inflamatoria materna que es muy característica en la fisiopatología de esta afección. La preeclamsia comprende la activación del sistema de coagulación. Se piensa que la disfunción endotelial se relaciona con una respuesta inflamatoria y esta desempeña una característica principal en el inicio temprano y grave de preeclamsia. Se plantea que la dieta materna tiene mucha influencia en el desarrollo de un trastorno hipertensivo en el embarazo, por ejemplo, una dieta caracterizada por un consumo de verduras, frutas y aceites vegetales se relacionan con un riesgo reducido de preeclampsia [22].

Menon y Fortunato manifiestan en su revisión que los probióticos son microorganismos vivos que cuando son administrados en cantidades correctas dan un beneficio adicional para la salud del huésped. Los prebióticos cuando son ingeridos por vía oral tienen una mayor probabilidad de colonizar la vagina y normalizar la flora bacteriana en el tracto genital inferior, los probióticos pueden tener un efecto antinflamatorio de los liposacaridos en las células del trofoblasto placentario humano, un estudio dio a conocer que las madres que se les administro leche que contenían probióticos durante la primera mitad del embarazo presentaban un riesgo reducido de preeclampsia [23].

Halkjaer y sus colaboradores en su artículo de revisión afirman que el consumo de probióticos puede aminorar el riesgo de preeclampsia, modulando la presión arterial. Esto serviría como hallazgo potencial al tratar de explicar y entender la asociación que existe entre el consumo de probióticos y la preeclampsia, especialmente cuando los probióticos se consumen al final del embarazo o si la ingesta durante el embarazo temprano fue certera, dará como resultado un menor riesgo de parto prematuro [5, 24].

Según Sohn uno de los papeles fundamentales que proporciona la administración de los probióticos durante el embarazo es la de protección frente a la preeclampsia, diabetes gestacional, infecciones vaginales, aumento de peso materno, infantil, y posteriores. Adicionalmente, de su seguridad, se ha demostrado también que su uso reduce la proteína $C$ reactiva, que es un reactante de fase aguda, que se asocia con efectos adversos cuando ocurren afecciones maternas como es la preeclampsia y diabetes gestacional. Si se consume una mezcla de probióticos en las etapas finales del embarazo también ocurre la modulación en las citoquinas antiinflamatorias en especial la interleucina (IL)-4 e IL-10 disminuyendo por ende en gran medida estas [25].

En otro estudio se destaca que la ingesta de la leche con probióticos fue más frecuente en un grupo de mujeres mayores, primíparas que se encontraban en un rango de IMC $<25$, las cuales no fumaban, usaban suplementos dietéticos, consumían yogurt y presentaban un nivel de educación alto acompañado con los ingresos familiares. Entre ellas se estimó un promedio de 1,5 a 2 tazas leche con probióticos durante la etapa de embarazo especialmente en el tercer trimestre lo cual proporcionó un menor riesgo de padecer preeclampsia y en aquellas que cursaban con un embarazo tardío esto 
tuvo una repercusión muy importante al disminuir la probabilidad de tener un parto prematuro ya sea espontaneo y parto prematuro iatrogénico [26].

Para Karen y sus colaboradores, los probióticos tienen un efecto sobre la cascada inflamatoria cuando sucede una posible infección, por este motivo podría ocurrir un parto prematuro; el efecto de la leche probiótica sobre la preeclamsia y parto prematuro también tuvo mucha relación con la FFQ (Frecuencia de Consumo de Alimentos) a mediados del embarazo, teniendo siempre en cuenta factores ambientales, infecciones y consumo de medicamentos que también pueden influir [19]. Se debe tener en cuenta que las mujeres al estar embarazas tienden a cambiar las conductas alimenticias y se debería aprovechar aumentando el consumo de los probióticos de mejor manera si se lo hace en el primer trimestre del embarazo, sin embargo algunas mujeres disminuyen el consumo de comida durante el segundo y tercer trimestre del embarazo, cuando presentan los síntomas más fuertes como náuseas y vómitos, esto hace pensar que el consumo de probióticos tiene mucha relación cuando se consume durante diferentes puntos de tiempo en el embarazo; de manera profiláctica resulta de mayor beneficio el consumo de leche con probióticos y se recomienda ingerir también antes del embarazo logrando la disminución del índice de infecciones y el aumento de la inmunidad materna ya que tienen el potencial de impactar el estado fisiopatológico como los procesos implicados en la hipertensión, inflamación, funciones renales y diabetes [27, 28].

Otro estudio que duro aproximadamente 9 semanas, en un total de 70 mujeres, se midió el estrés oxidativo y el aumento de los niveles de GR (Glutatión Reductasa) después del consumo de yogurt con probiótico, fue positivo con lo cual se corrobora de nuevo la importancia de reducir el estrés oxidativo, hay que recordar el hecho de que la placenta es rica en mitocondrias y se requiere mayor cantidad de oxígeno durante el tercer trimestre, por consiguiente, esta etapa del embarazo es la que se encuentra relacionada con niveles altos de estrés [29].

Entre el estrés oxidativo y disfunción endotelial ocurre una asociación que cuando se generaliza, se cree que puede ser la causa del aparecimiento de la preeclamsia, peso neonatal bajo, parto prematuro, restricción del crecimiento fetal, eclampsia, infecciones, desnutrición materna y diabetes. Los probióticos al ser bacterias vivas y al consumir con frecuencia mejoran el estrés oxidativo al reducirlo y a la vez aumentar los niveles de glutatión. Los rangos de edad en el estudio realizado cursaron entre 24 y 25 años y con un rango de peso de 68 a $71 \mathrm{~kg}$ correspondiendo a un IMC de 28 y 29, en ellas se encontró que al haber consumido yogurt con probióticos en especial los que contienen

L. acidophilus LA5 y B. lactis BB12 se generó un aumento de los niveles de GR (Glutatión Reductasa), lo cual fue beneficioso puesto que esta enzima juega un papel importante en la defensa de diferentes tejidos y órganos en la fisiopatología de varias enfermedades; este estudio también se encontró la relación con la proteína $C$ reactiva como biomarcador de alta sensibilidad que como otras investigaciones afirman que puede disminuir los niveles [30, 31]. 


\section{Conclusiones}

La finalidad de los probióticos es mantener o mejorar el microbiota normal del individuo, actuales estudios muestran evidencia que emplear alimentos que contengan probióticos, ayuda a la reducción de la respuesta inflamatoria por lo cual disminuye la probabilidad de padecer preeclampsia, que es la complicación más común en una mujer embarazada después de la semana 20 de gestación, posterior a una insuficiente oxigenación que genera un estado de estrés oxidativo por la deficiencia de perfusión del trofoblasto. Para que el mecanismo realice su efecto preventivo, es necesario que se administre principalmente en el segundo y tercer trimestre de embarazo, aunque el consumo de probióticos con anterioridad a estos, resulta de mucho mayor beneficio, en especial si se consume en combinación de varias cepas de probióticos. Su mecanismo de acción consiste en mantener la integridad del epitelio particularmente intestinal o bien facilitando la reparación; ayuda al sistema inmunológico, al activar los macrófagos locales que presentan antígenos a los linfocitos $B$ y producen inmunoglobulina A secretora local y sistémica, y a la vez disminuye la proteína $C$ reactiva. Además de prevenir la preeclampsia puede reducir el riesgo a infecciones vaginales, ya que producen ácido láctico, el mismo que crea un ambiente inhóspito para bacterias.

\section{Agradecimientos}

Los autores agradecemos formalmente a la Dirección de Bibliotecas y Recursos para el Aprendizaje y la Investigación, por la asesoría brindada para la búsqueda técnica en las bases de datos.

\section{Conflicto de Intereses}

Los autores del siguiente artículo, expresan no poseer ningún conflicto de interés en la realización del trabajo de investigación.

\section{Limitación de Responsabilidad}

Toda la información proporcionada en la siguiente revisión bibliográfica son absoluta responsabilidad de los autores legítimos.

\section{Fuentes de Apoyo}

Propias del grupo de autores de este artículo.

\section{References}

[1] Alkema L, Chou D, Hogan D, Zhang S, Moller ABGA. Global, regional, and national levels and trends in maternal mortality between 1990 and 2015, with scenario-based projections to 2030: A systematic 
analysis by the UN Maternal Mortality Estimation Inter-Agency Group. Lancet. 2016;17(287):462-74.

[2] Ronsmans CGW. Maternal survival series steering group, "maternal mortality; who, when, where and why." Lancet. 2006;1(23-3).

[3] Villar J, Say L, Gulmezoglu AM, et al. L. Eclampsia and pre-eclampsia: A health problem for 2000 years. Lancet. 2003;3:189-207.

[4] Godoy TGM, Mabel ZJ. Restricción de crecimiento intrauterino: Causas, características clínicas, y evaluación de factores asociados a policitemia sintomática. Rev. chil. pediatr. 2010;81(4):366-367.

[5] Organizacion Panamericana de la Salud. Recomendaciones de la OMS en la prevencion y tratamiento de preclampsia y eclampsia. 2019. Available from: https://www.paho.org/clap/index.php?option=com content\&view=article\&id=570:dia-de-concientizacion-sobre-la-preeclampsia-22-de-mayo\&ltemid= 215\&lang=es

[6] Martín L, Carbajal G. Actualización en la fisiopatología de la preeclampsia. Scielo Rev chil Obs ginecol. 2014;60(2304-5132):6.

[7] HVMV, AGA, EMAM. Preeclampsia un problema de salud pública mundial. Scielo Rev chil Obs ginecol. 2012;77(6):471-6.

[8] Organización Panamericana de la Salud. Guias de Manejo de las Complicaciones obstetricas. Minist Salud Panama; 2009.

[9] Carnicé RT. Probióticos. Concepto y mecanismos de acción. An Pediatr. 2006;4(1):30-41.

[10] Mayo Clinic. ¿Qué son los probióticos y los Prebióticos? Mayo Foundation for Medical Education and Research; 2019.

[11] Acton A. Preeclampsia: Nex insights for the healthcare professional. ScholarlyBrief. 2011. Available from: https://books.google.com.ec/books?id=b-o6rDwR7qsC\&printsec=frontcover\&dq=preeclampsia\&hl= es-419\&sa=X\&ved=0ahUKEwj4tZCS-OHnAhWhmOAKHYnpDhQQ6AEIKjAA\#v=onepage\&q\&f=false

[12] Herna AS. Celulas colaboradoras artritis reumatoide. Reumatologia Clin. 2009;5:1-5.

[13] Marteau P, Vaerman JP, Dehennin JP, Bord S, Brassart D PP. Effects of intrajejunal perfusion and chronic ingestion of Lactobacillus johnsonii strain La1 on serum concentrations and jejunal secretions of immunoglobulins and serum protein in healthy humans. Gastroenterol Clin Biol. 1997;4(293):8.

[14] Ogawa T, Asai Y, Tamai R, et al. Natural killer cell activities of synbiotic Lactobacillus casei ssp. case in conjunction with dextran. Clin Exp Immunol. 2005;103-9.

[15] Myhre R., Brantsæter AL, Myking S, et al. La ingesta de alimentos probióticos y el riesgo de parto prematuro espontáneo. Clin Nutr. 2011;93.

[16] Nordqvist M, Jacobsson B BA-L. Timing of probiotic milk consumption during pregnancy and effects on the incidence of preeclampsia and preterm delivery: a prospective observational cohort study in Norway. BMJ Open. 2018.

[17] Garrote A, Bonet YR. Probioticos. Elsevier. 2017;31:13-6.

[18] Pradenas DM. Infecciones cérvico vaginales y. Genit Infect pregnancy. 2014;25(6):925-35.

[19] Vizcaíno R, Macias-Tomei C, Márquez SJC, Morales ATN. Usos clínicos de los probióticos. Scielo Rev chil Obs ginecol. 2016;1:029-040.

[20] Escobar L. Anestesia Local y locorregional en cirugia menor. Reveure. 2006;32(2):73-98.

[21] Ministerio de Salud Pública. Trastornos hipertensivos del embarazo. Guía de Practica Clínica (GPC). 2016;2.

[22] Alexandra L, Villamil T, Carolina A, et al. Cuidado nutricional en la prevención de la preeclampsia: Una revisión sistemática Introducción Material y métodos. 2018;24(2).

[23] Aguiló IP. Las bacterias contraatacan. Not Segur. 2017;6(2):1-27.

[24] Halkjaer S, Nilas L, Carlsen E, Cortes D, Halldórsson T, Pedersen AKKT. Effects of probiotics (Vivomixx ${ }^{\circledR}$ ) in obese pregnant women and their newborn: study protocol for a randomized controlled trial. Pubmed. 2016;17:491.

[25] Sohn K, Underwood MA. Prenatal and postnatal administration of prebiotics and probiotics. Seminars in Fetal \& Neonatal Medicine. 2017.

[26] Zatollah A, Samimi M, Tabasi Z, Talebian P, Azarbad Z, Esmaillzadeh A. Effect of daily consumption of probiotic yoghurt on lipid profiles in pregnant women: a randomized controlled clinical trial. [Internet]. 2018;25(9):1552- 1556.

[27] Lindsay KL, Walsh CA, Brennan L, McAuliffe FM. Probiotics in pregnancy and maternal outcomes: A systematic review. The Journal of Maternal-Fetal \& Neonatal Medicine. 2013;26(8):772-778.

[28] Doege K, Garjecki D, Zyriax B-C, et al. Impact of maternal supplementation with probiotics during pregnancy on atopic eczema in childhood - a meta-analysis. Br J Nutr [Internet]. 2012;107:1-6.

[29] Badehnoosh B, Karamali M, Zarrati M, et al. The effects of probiotic supplementation on biomarkers of inflammation, oxidative stress and pregnancy outcomes in gestational diabetes. The Journal of Maternal-Fetal \& Neonatal Medicine. 2017. 
[30] Jamilian M, Bahmani F, Vahedpoor Z, et al. Effects of probiotic supplementation on metabolic status in pregnant women: A randomized, double-blind, placebo-controlled trial. Arch Iran Med. 2016;19:687-2.

[31] Olveira G, González-molero I. Endocrinología y nutrición actualización de probióticos, prebióticos y simbióticos. Elsevier. 2016;63(9):482-94.

[32] Pagnini C, Saeed R, Bamias G, Arseneau KO, Pizarro TT C. Probiotics promote gut health through stimulation of epithelial innate immunity. Proc Natl Acad. 2010;1(454):9. 